- 研究报告・

\title{
长江上游特有种长鯺吻鮈种群数量和资源利用评估
}

\author{
熊 飞 $1^{1^{*}}$ 刘红䎦 ${ }^{1}$ 段辛斌 $^{2^{*}}$ 刘绍平 ${ }^{2}$ 陈大庆 ${ }^{2}$ \\ 1 (江汉大学生命科学学院, 武汉 430056) \\ 2 (中国水产科学研究院长江水产研究所, 武汉 430223)
}

\begin{abstract}
摘要: 长鳍吻鮈(Rhinogobio ventralis) 为长江上游特有种, 由于过度捕捞和大坝建设, 其种群生存受到极大威胁。 为了解三峡工程蓄水后、金沙江一期工程蓄水前该物种的种群动态, 作者利用2007-2009年长江上游江津和宜宾江 段调查获取的体长频率数据, 评估了其生长和死亡参数、种群数量及资源利用情况。结果表明, 长江上游长鰽吻 鮈渔获群体体长范围为 $52-250 \mathrm{~mm}$, 体重范围为 $2.7-307.2 \mathrm{~g}$, 平均体长为 $150.8 \pm 40.7 \mathrm{~mm}$, 平均体重为 $72.3 \pm 49.7$ $\mathrm{g}$ 。江津江段长鰙吻鮈平均体长 $(168.6 \pm 29.5 \mathrm{~mm})$ 显著大于宜宾江段 $(125.6 \pm 41.2 \mathrm{~mm})$ 。长鲧吻鮈体长 - 体重关系为: $W=6.06 \times 10^{-6} L^{3.20}\left(R^{2}=0.98, P<0.01, n=436\right)$ 。由体长频率法拟合出渐近体长 $\left(L_{\infty}\right)$ 为 $338 \mathrm{~mm}$, 生长系数 $(k)$ 为 $0.24 / \mathrm{yr}$ 。由Pauly公式、Gunderson和Dygert公式、Jensen公式等 3 种方法估算出其平均自然死亡系数为 0.43 。由长度 转渔获物曲线估算出江津和宜宾江段长鯺吻鮈的总死亡系数分别为 2.26 和 2.09 。江津和宜宾江段长鰙吻鮈资源开 发率分别为 0.81 和 0.79 , 已超过由Beverton-Holt动态综合模型估算出的最大开发率 0.57 和 0.62 。由体长实际种群分 析估算出 $2007 、 2008$ 和 2009 年江津江段长鯺吻鮈种群数量分别为 $68,247 、 67,432$ 和 176,266 尾, 平均为 103,982 尾; 宜 宾江段种群数量分别为 22,953 尾、46,340尾和 34,021 尾，平均为 34,438 尾，表明江津江段种群数量高于宜宾江段。 长鯺吻鮈资源已被过度开发, 建议加强种群动态监测, 延长禁渔期及开展栖息地修复等措施保护这一特有物种。 关键词: 种群参数; 生长; 死亡; 种群数量; 资源评估; 长鯺吻鮈
\end{abstract}

\section{Estimating population abundance and utilization of Rhinogobio ventralis, an endemic fish species in the upper Yangtze River}

\author{
Fei Xiong ${ }^{1 *}$, Hongyan Liu ${ }^{1}$, Xinbin Duan ${ }^{2 *}$, Shaoping Liu ${ }^{2}$, Daqing $\mathrm{Chen}^{2}$ \\ 1 School of Life Sciences, Jianghan University, Wuhan 430056 \\ 2 Yangtze River Fisheries Research Institute, Chinese Academy of Fishery Sciences, Wuhan 430223
}

\begin{abstract}
Rhinogobio ventralis, an endemic species in the upper Yangtze River, has been threatened by overfishing and dam construction. To understand its population dynamics after the Three Gorges Reservoir was built and before the Xiangjiaba and Xiluodu reservoirs were built, we employed body length-frequency data to estimate growth and mortality parameters, population abundance and utilization of the fish based on surveys in the Jiangjin and Yibin sections of the upper Yangtze River conducted from 2007-2009. Fish ranged from 52 to $250 \mathrm{~mm}$ in length and 2.7 to $307.2 \mathrm{~g}$ in weight, with an average length of $150.8 \pm 40.7 \mathrm{~mm}$ and an average weight of $72.3 \pm 49.7 \mathrm{~g}$. The average size of the fish in the Jiangjin section of the Yangtze River $(168.6 \pm 29.5 \mathrm{~mm})$ was significantly larger than that in Yibin section $(125.6 \pm 41.2 \mathrm{~mm})$. The length-weight relationship of $R$. ventralis was well-fit with a power function, $W=6.06 \times 10^{-6} L^{3.20}\left(R^{2}=0.98\right.$, $P<0.01, n=436)$. Asymptotic length $\left(L_{\infty}\right)$ and growth constant $(k)$ were estimated using length frequency data as $338 \mathrm{~mm}$ and $0.24 / \mathrm{yr}$, respectively. Average natural mortality was comprehensively estimated as 0.43 using three empirical formulas proposed by Pauly, Gunderson \& Dygert, and Jensen, respectively. The total mortalities were estimated using a length-converted catch curve analysis as 2.26 in the Jiangin section and 2.09 in the Yibin section of the Yangtze River. The observed exploitation rates were 0.81 in the Jiangjin section and 0.79 in the Yibin section, which were higher than the maximum exploitation rates of 0.57 in the Ji-
\end{abstract}

收稿日期: 2015-10-09; 接受日期: 2015-12-11

基金项目: 国家自然科学基金(51109091 和 51310105036)

* 共同通讯作者 Co-authors for correspondence. E-mail: xf9603@163.com,duan@yfi.ac.cn 
angjin section and 0.62 in the Yibin section that were estimated using Beverton \& Holt $Y^{\prime} / R$ analysis. Population abundance of $R$. ventralis in the Jiangjin section was estimated by length-structured virtual population analysis as 68,247 in 2007, 67,432 in 2008, and 176,266 in 2009, respectively, with an average of 103,982; while abundance in the Yibin section was estimated as 22,953 in 2007, 46,340 in 2008, and 34,021 in 2009 , respectively, with an average of 34,438 , indicating the population abundance was higher in the Jiangjin section than the Yibin section. We conclude that overfishing is occurring in these stocks of $R$. ventralis and recommend a longer period of monitoring in order to understand population dynamics of $R$. ventralis. We further suggest that management authorities establish a longer closed period for fishing and undertake habitat restoration to protect the endemic fish species.

Key words: population parameters; growth; mortality; population abundance; stock assessment; Rhinogobio ventralis

生境破碎化是物种退化和生物多样性丧失的 重要原因(Fahrig, 2003; 蒋志刚和葛颂, 2005)。越来 越多的物种因人类的扩张而被迫生活于破碎、萎缩 的生境中, 其种群参数、种群数量及种群生存力等 也随之改变(Letcher et al, 2007; Morita et al, 2009)。 河流水利工程的建设, 尤其是梯级开发, 损害了河 流的连续性, 造成水域生境破碎化, 使物种迁移、 扩散和交流受阻, 生物多样性下降(Esguicero \& Arcifa, 2010)。长江上游密集的水利梯级开发对特有 鱼类的影响受到广泛关注(Xie, 2003; Gao et al, 2010)。

长鯺吻鮈 (Rhinogobio ventralis)隶属于鲤科鮈 亚科吻鮈属(Rhinogobio), 是长江上游特有种, 也是 该江段重要的渔获对象。该物种为典型的流水性种 类, 产漂流性卵, 对栖息生境面积要求较大(周启贵 和何学福, 1992)。三峡水库蓄水后, 长江上游约600 $\mathrm{km}$ 干流江段形成河谷型水库, 原来栖息于此的长 鯺吻鮈等特有鱼类因其栖息生境大范围萎缩将逐 渐从库区消失(Park et al, 2003), 而宜宾江段以上的 向家坝、溪洛渡等金沙江一期工程的建设, 将加剧 这种影响(陈大庆等, 2005)。随着坝下水文和水温条 件的改变, 坝下鱼类的繁殖活动也将受到影响(段 辛斌等, 2015), 长江上游长鯺吻鮈种群生存受到严 重威胁(刘军, 2004)。

关于长鯺吻鮈的研究, 主要集中在年龄、生长 和繁殖等方面(段中华等, 1991; 邓辉胜和何学福, 2005; 鲍新国等, 2009), 而关于其种群数量和渔业 资源利用方面的关注相对较少。张松(2003) ${ }^{1}$ 和刘军 等(2010)曾根据体长股方法分别估算了三峡水库蓄 水前合江和宜宾江段长鯺吻鮈的种群数量。江津江

(1) 张松 (2003) 长江上游合江江段渔业现状评估及长鯺吻鮈的资源评 估.硕士学位论文, 华中农业大学, 武汉.
段位于三峡水库库尾以上, 是鱼类在长江上游和三 峡库区之间交流的重要生态通道，长鯺吻鮈为该江 段的优势种(熊飞等，2014); 宜宾江段位于向家坝 工程以下，长鯺吻鮈为该江段的亚优势种。三峡水 库蓄水后, 江津和宜宾江段长鯺吻鮈的种群动态颇 受关注。

鱼类种群参数和种群数量是渔业资源评估、管 理的重要依据。以往对鱼类种群参数的估算主要依 据鱼类的年龄-体长数据, 因此需要对大量标本进 行年龄鉴定(詹秉义, 1995)。而直接利用基于时间序 列的体长频率数据来估算种群参数和种群数量可 以避免年龄鉴定，提高渔业资源评估的精度和效率 (Ghosh et al, 2012; Wang et al, 2012; Johnson \& Tamatamah, 2013), 但该方法在我国淡水鱼类方面的 应用还比较缺乏( 吴斌等, 2013)。本研究根据 2007-2009年长江上游江津和宜宾江段的渔业调查 数据, 利用基于时间序列的体长频率数据分析方法 对长鯺吻鮈的生长和死亡参数、种群数量及资源利 用情况进行评估，以期了解三峡工程蓄水后、金沙 江一期工程蓄水前该物种的种群动态, 为长江上游 特有鱼类保护提供依据, 为大型水利工程的生态学 效应评估积累资料。

\section{1 材料与方法}

\section{1 数据来源}

数据来源于2007-2009年对长江上游江津和宜 宾干流江段的渔业资源调查，调查时间主要集中在 每年的5-7月及9-11月，每个月每个江段的调查时 间根据渔获物多少决定，一般持续7-10天。江津江 段调查范围为朱杨镇上游至石门镇约 $15 \mathrm{~km}$ 江段, 宜宾江段调查范围为打渔村至新村约 $15 \mathrm{~km}$ 江段 (图1)。调查渔具主要包括流刺网(网目3-6 cm)、百 


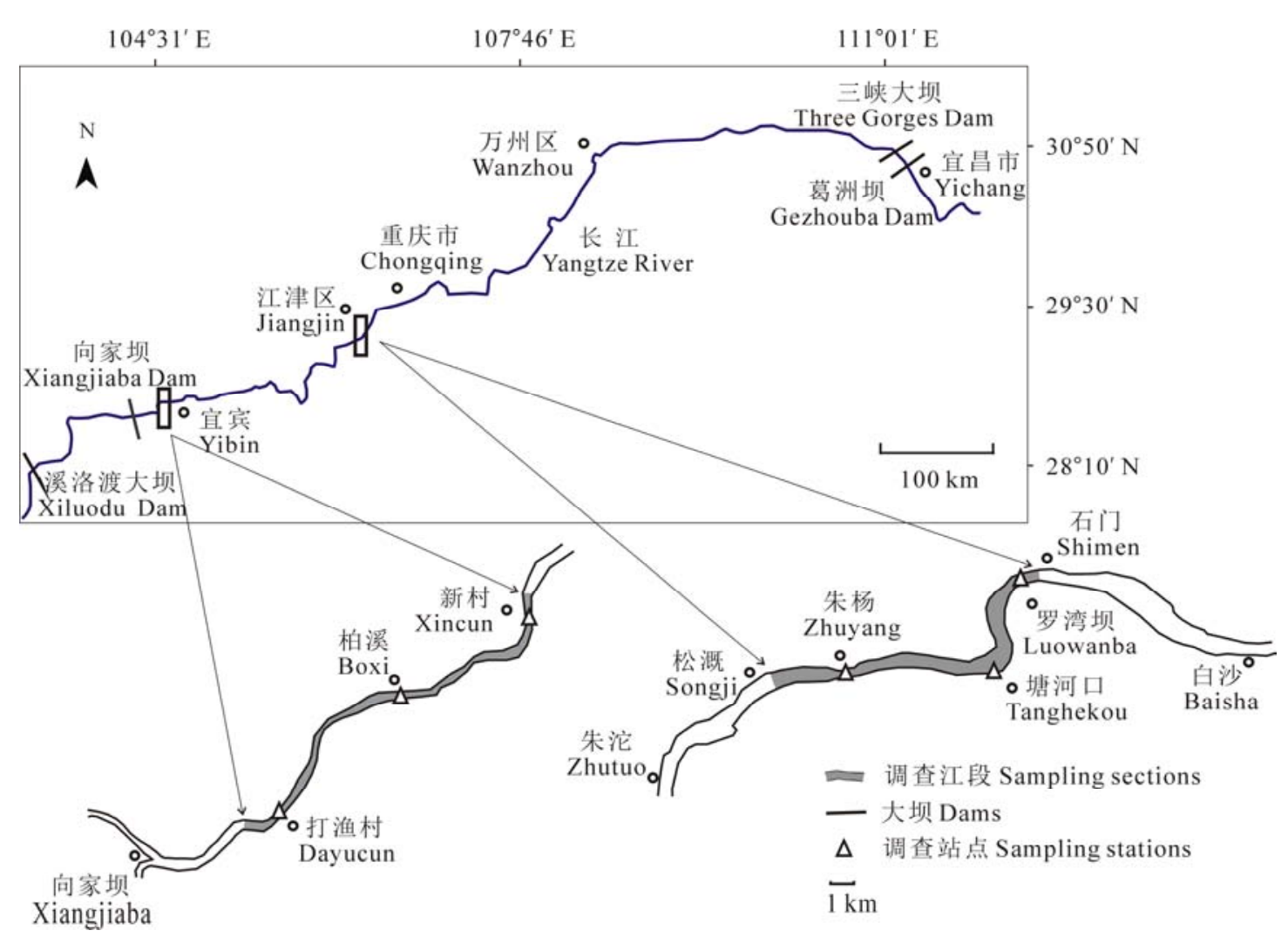

图1 长江上游调查站点设置

Fig. 1 Location of sampling stations in the upper Yangtze River

袋网(网目 $2 \mathrm{~cm}$ )和小钩。每种渔具选取 3 条固定渔船 进行渔获物调查, 并对其他渔船进行不定期抽查, 从渔船收集所有渔获物后进行分类、计数和称重。 物种鉴定主要参考《四川鱼类志》(丁瑞华, 1994)。 测量长鯺吻鮈新鲜个体体长 $(L)$ 、全长 $(T L)$ 和体重 $(W)$, 体长为吻端到尾部前端最后一枚鳞片的距离, 全长为吻端到尾鯺末端的距离。体长精确到 $1 \mathrm{~mm}$, 体重精确到 $1 \mathrm{~g}$ 。对调查江段的作业渔船数量及单位 捕捞努力量渔获量进行统计。根据作业江段捕捞的 平均强度, 流刺网和百袋网每天作业网次按 10 网的 标准计算单位捕捞努力量渔获量。小钩作业时间为 $12 \mathrm{~h}, 18: 00$ 放钩, 次日6:00收钩。在两个江段共统计 渔获物 $1,292 \mathrm{~kg}$, 合计 16,980 尾, 对436尾长鲔吻鮈 进行了生物学测量, 其中江津江段256尾, 宜宾江 段180尾。

\section{2 参数估算}

体长-体重关系依据Keys (1928)公式 $W=a L^{b}$ 进 行拟合, 其中 $a, b$ 为常数, $a$ 为生长的条件因子, $b$ 为 幂指数系数。用von Bertalanffy生长方程描述长鯺吻
鮈的生长规律, 渐近体长 $\left(L_{\infty}\right)$ 和生长系数 $(k)$ 利用基 于体长频率数据的 Powell-Wetherall plot 法和 ELEFAN I法拟合, 总死亡系数 $(Z)$ 由体长转渔获物 曲线 (converted-length catch curve)进行估算(Gayanilo et al, 2005)。理论生长起点年龄 $\left(t_{0}\right)$ 依Pauly (1979)经验公式计算:

$$
\begin{aligned}
& \log \left(-t_{0}\right)=-0.3922-0.2752 \log L_{\infty}-1.038 \log k(1) \\
& \text { 自然死亡系数 }(M) \text { 依Pauly (1980)、Gunderson和 }
\end{aligned}
$$
Dygert (1988)和Jensen (1996)等3种方法进行估算, 然后取平均值:

$$
\begin{aligned}
& \log M=-0.0066-0.279 \log L_{\infty}+0.6543 \log k+ \\
& 0.4634 \log T \\
& M=0.03+1.68 G S I \\
& M=1.65 / X_{\mathrm{m}}
\end{aligned}
$$

式中, $L_{\infty}$ 表示渐进全长, 单位为 $\mathrm{cm}$, 依据拟合的体 长-全长关系, 将渐近体长换算成渐近全长。 $T$ 表示 栖息江段的平均水温, 取值 $18.4^{\circ} \mathrm{C}$ (田辉伍等, 2013); GSI和 $X_{\mathrm{m}}$ 分别代表雌性个体的最大性成熟系 数和性成熟年龄, 分别取值 $10.7 \%$ 和 3 (周启贵和何 
学福, 1992)。

捕捞死亡系数:

$F=Z-M$

资源开发率:

$E=F / Z$

采用Beverton-Holt动态综合模型相对单位补充 量产量(生物量) $\left(Y^{\prime} / R, B^{\prime} / R\right)$ 曲线评价资源利用情况 (Gayanilo et al, 2005), 模型中 $E_{\max }$ 表示最大开发率, 即能获得最大产量时的开发率, $E_{10}$ 表示“最适”开发 率, 即资源群体边际产量减少到理论原始资源边际 产量 $1 / 10$ 时的开发率, $E_{50}$ 表示资源量下降到原始水 平 $50 \%$ 时的开发率。

\section{3 种群数量估算}

根据不同渔具的年总渔获量及长鯺吻鮈在渔 获物中的比例计算出长鯺吻鮈的年总渔获量 $(Y)$ :

$$
\begin{aligned}
& Y=\sum\left(Y_{\mathrm{i}} \times P_{\mathrm{i}}\right) \\
& Y_{\mathrm{i}}=x_{\mathrm{i}} \times f_{\mathrm{i}} \times t_{\mathrm{i}}
\end{aligned}
$$

式中, $\mathrm{i}$ 表示不同渔具, $P_{\mathrm{i}}$ 为长鰙吻鮈在 $\mathrm{i}$ 渔具渔获物 中的比例, $Y_{\mathrm{i}}$ 为 $\mathrm{i}$ 渔具的年总渔获量, $x_{\mathrm{i}}$ 为 $\mathrm{i}$ 渔具单位捕 捞努力量渔获量(尾/(船. 天) $), f_{\mathrm{i}}$ 为渔具 $\mathrm{i}$ 的捕捞努力 量, 即作业渔船数量。江津江段作业渔船8条, 其中 流刺网渔船 5 条, 百袋网渔船 3 条; 宜宾江段作业渔 船 15 条, 其中流刺网渔船 12 条, 小钩渔船 3 条。 $t_{\mathrm{i}}$ 为 渔具 $i$ 的年作业时间(天), 除去春季禁渔时期(2-4月) 和洪水季节(7-8月)等，按200天计算。

采用FiSATII软件中的体长结构实际种群分析 (length-structured virtual population analyses)估算种 群数量(Gayanilo et al, 2005)。最大体长组捕捞死亡 系数 $\left(F_{\mathrm{t}}\right)$ 初始值取 0.5 , 通过迭代法确定最终值 (吴斌 等, 2013)。此外, 根据最大体长组资源开发率 $\left(E_{\mathrm{t}}\right)$ 确 定 $F_{\mathrm{t}}$, 估算在 $E_{\mathrm{t}}$ 取值 0.5 和 0.8 时的种群数量, 比较 2 种方法确定 $F_{\text {t }}$ 值情况下的种群估算结果差异。

\section{2 结果}

\section{1 渔获群体结构}

长江上游长鳍吻鮈渔获群体体长范围为 $52-$ $250 \mathrm{~mm}$, 平均体长为 $150.8 \pm 40.7 \mathrm{~mm}$, 体重范围为 2.7-307.2 g, 平均体重为 $72.3 \pm 49.7 \mathrm{~g}$ 。江津江段优 势体长组为 $135-210 \mathrm{~mm}$, 约占个体数量的 $84.4 \%$, 其中 $165-195 \mathrm{~mm}$ 体长组中的个体约占 $56.3 \%$ 。宜宾 江段优势体长组为75-120 mm 和150-180 mm, 分别 占 $53.9 \%$ 和 $29.4 \%$ (图2)。江津江段长鯺吻鮈平均个
体体长 $(168.6 \pm 29.5 \mathrm{~mm})$ 显著大于宜宾江段(125.6 \pm $41.2 \mathrm{~mm})(F=12.0, d f=435, P<0.001)$ 。

\section{2 生长参数}

长江上游长鯺吻鮈体长 $(L)$ 与全长 $(T L)$ 呈显著 线性关系: $T L=0.685+1.182 L\left(R^{2}=0.99, P<0.01\right.$, $n=436)$ 。体长 $(L)$ 与体重 $(W)$ 呈显著幂函数关系(图 3): $W=6.06 \times 10^{-6} L^{3.20}\left(R^{2}=0.98, P<0.01, n=436\right)$ 。 其中, 江津江段体长-体重关系为: $W=7.02 \times$
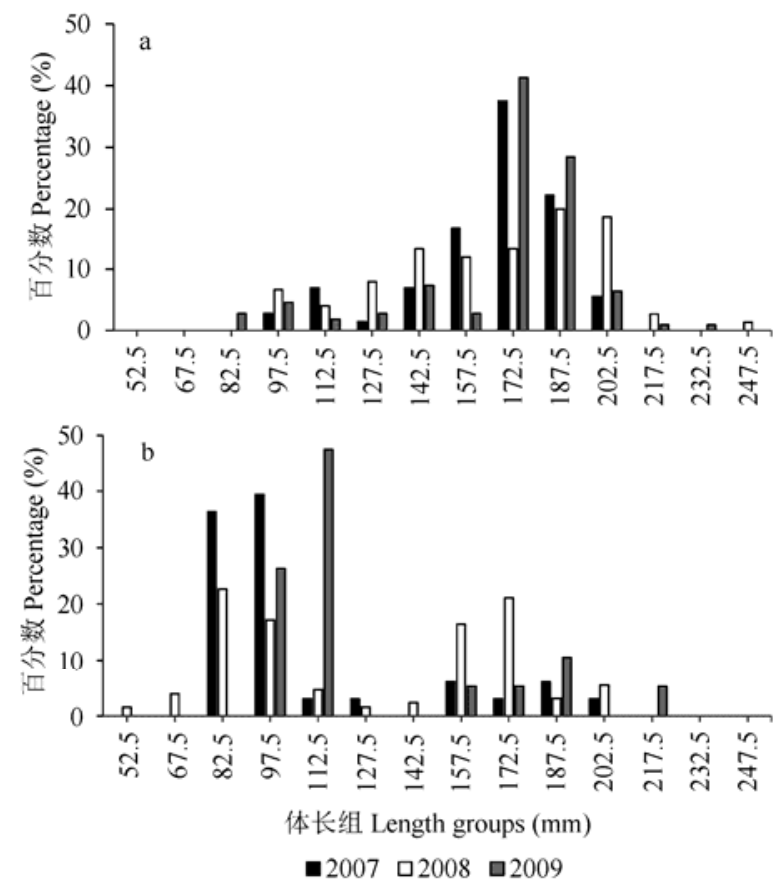

图2 江津(a)和宜宾(b)江段长鯺吻鮈体长结构

Fig. 2 Length structure of Rhinogobio ventralis in the Jiangjin (a) and Yibin (b) sections

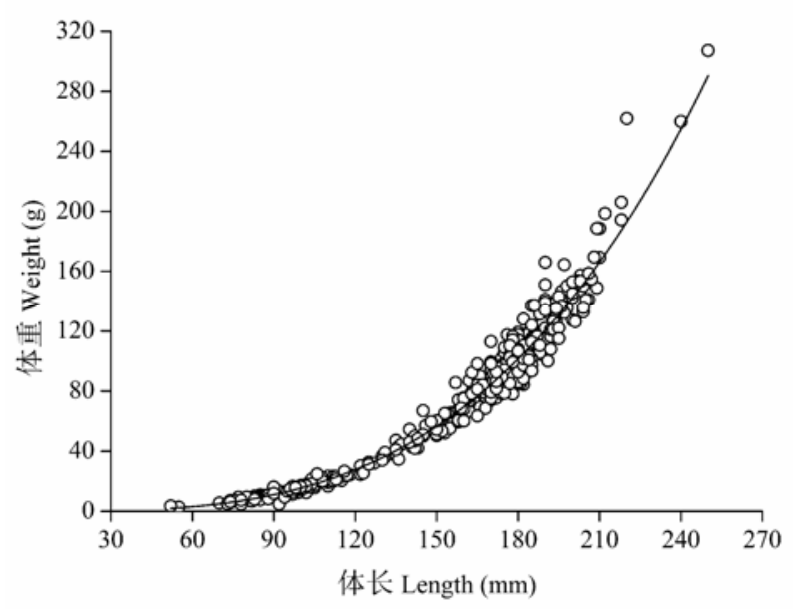

图3 长江上游长鯺吻鮈体长-体重关系

Fig. 3 Length-weight relationship of Rhinogobio ventralis in the upper Yangtze River 
$10^{-6} L^{3.18}\left(R^{2}=0.97, P<0.01, n=256\right)$; 宜宾江段体 长一体重关系为: $W=7.51 \times 10^{-6} L^{3.15}\left(R^{2}=0.98, P<\right.$ $0.01, n=180$ )。

由体长频率法拟合出长鳍吻鮈的生长参数 $L_{\infty}=$ $338 \mathrm{~mm}, k=0.24 / \mathrm{yr}$, 此时 $t_{0}=-0.83$ 。拐点年龄为 4.02龄, 对应的体长和体重分别为 $232.4 \mathrm{~mm}$ 和 226.1 $\mathrm{g}$ 。长鯺吻鮈体长和体重von Bertalanffy生长方程为:

$$
\begin{aligned}
& L_{\mathrm{t}}=338\left[1-e^{-0.24(t+0.83)}\right] \\
& W_{\mathrm{t}}=749.9\left[1-e^{-0.24(t+0.83)}\right]^{3.20}
\end{aligned}
$$

\section{3 死亡系数和开发率}

由 3 种方法估算出长鯺吻鮈的自然死亡系数分 别为 $0.52 、 0.21$ 和 0.55 , 取平均值 $M=0.43$ 。长度转 渔获物曲线分析结果表明(表1), 江津和宜宾江段长 鯺吻鮈的总死亡系数、捕捞死亡系数和资源开发率 分别为 $Z=2.26 、 F=1.83, E=0.81$ 和 $Z=2.09 、 F=$ 1.66、 $E=0.79$ 。 Beverton-Holt动态综合模型的相对 补充量产量(生物量)曲线分析显示(图4), 江津江段 $E_{10}=0.46 、 E_{50}=0.32 、 E_{\max }=0.57$, 宜宾江段 $E_{10}=$ $0.50 、 E_{50}=0.35 、 E_{\max }=0.62$ 。江津和宜宾江段长鯺 吻鮈的资源开发率分别为 0.81 和 0.79 , 均已超过了 其最大开发率。

\section{4 种群数量}

渔获物分析结果表明，2007-2009年长鯺吻鮈 在江津江段流刺网中的数量比例分别为 $9.4 \% 、 6.9 \%$ 和 $12.9 \%$, 在百袋网中的比例分别为 $2.8 \% 、 0.7 \%$ 和 $1.1 \%$; 在宜宾江段流刺网中的比例分别为 $2.0 \%$ 、 $5.7 \%$ 和 $6.6 \%$, 在小钩中的比例分别为 $0 、 3.4 \%$ 和 $1.2 \%$ 。根据单位捕捞努力量渔获量和长鯺吻鮈的比 例估算出江津江段长鯺吻鮈2007-2009年的年总渔 获量分别为 $7,103 、 6,714$ 和 14,620 尾，年平均 9,479 尾; 宜宾江段长鯺吻鮈年总渔获量分别为 5,712 、 3,887 和 6,926 尾，年平均 5,508 尾。

由迭代法确定2007、2008和2009年江津江段最 大体长组的捕捞死亡系数 $F_{\mathrm{t}}$ 分别为 $0.38 、 0.35$ 和 0.32 ; 宜宾江段 $F_{\mathrm{t}}$ 分别为 $1.19 、 0.33$ 和 0.88 。体长结构实际 种群分析结果表明(图5), 2007-2009年江津江段长 鰙吻鮈种群数量分别为 68,247 尾(14.17 t)、67,432尾 $(15.55 \mathrm{t})$ 和 176,266 尾 $(29.36 \mathrm{t})$, 平均值为 103,982 尾 (19.69 t); 宜宾江段种群数量分别为 22,953 尾 (2.58 t)、 46,340 尾 $(5.15 \mathrm{t})$ 和 34,021 尾 $(6.37 \mathrm{t})$, 平均值为 34,438 尾(4.70 t)。

迭代法确定 $F_{\mathrm{t}}$ 值和直接由 $E_{\mathrm{t}}$ 确定 $F_{\mathrm{t}}$ 值 $\left(E_{\mathrm{t}}=0.5\right.$ 和
表1 2007-2009年长江上游长鯺吻鮈的死亡系数和开发率 Table 1 Mortalities and exploitation rates of Rhinogobio ventralis in the Jiangjin and Yibin sections from 2007-2009

\begin{tabular}{llllll}
\hline $\begin{array}{l}\text { 江段 } \\
\text { Section }\end{array}$ & 年份 Year & $Z$ & $M$ & $F$ & $E$ \\
\hline 江津 & 2007 & 3.01 & 0.43 & 2.58 & 0.86 \\
Jiangjin & 2008 & 1.90 & 0.43 & 1.47 & 0.77 \\
& 2009 & 2.53 & 0.43 & 2.10 & 0.83 \\
& 总体 Total & 2.26 & 0.43 & 1.83 & 0.81 \\
宜宾 & 2007 & 2.41 & 0.43 & 1.98 & 0.82 \\
Yibin & 2008 & 1.81 & 0.43 & 1.38 & 0.76 \\
& 2009 & 1.61 & 0.43 & 1.18 & 0.73 \\
& 总体 Total & 2.09 & 0.43 & 1.66 & 0.79 \\
\hline
\end{tabular}

Z: 总死亡系数; $M$ : 自然死亡系数; $F$ : 捕捞死亡系数; $E$ : 开发率 $Z$, Total mortality; $M$, Natural mortality; $F$, Fishing mortality; $E$, Exploitation rate
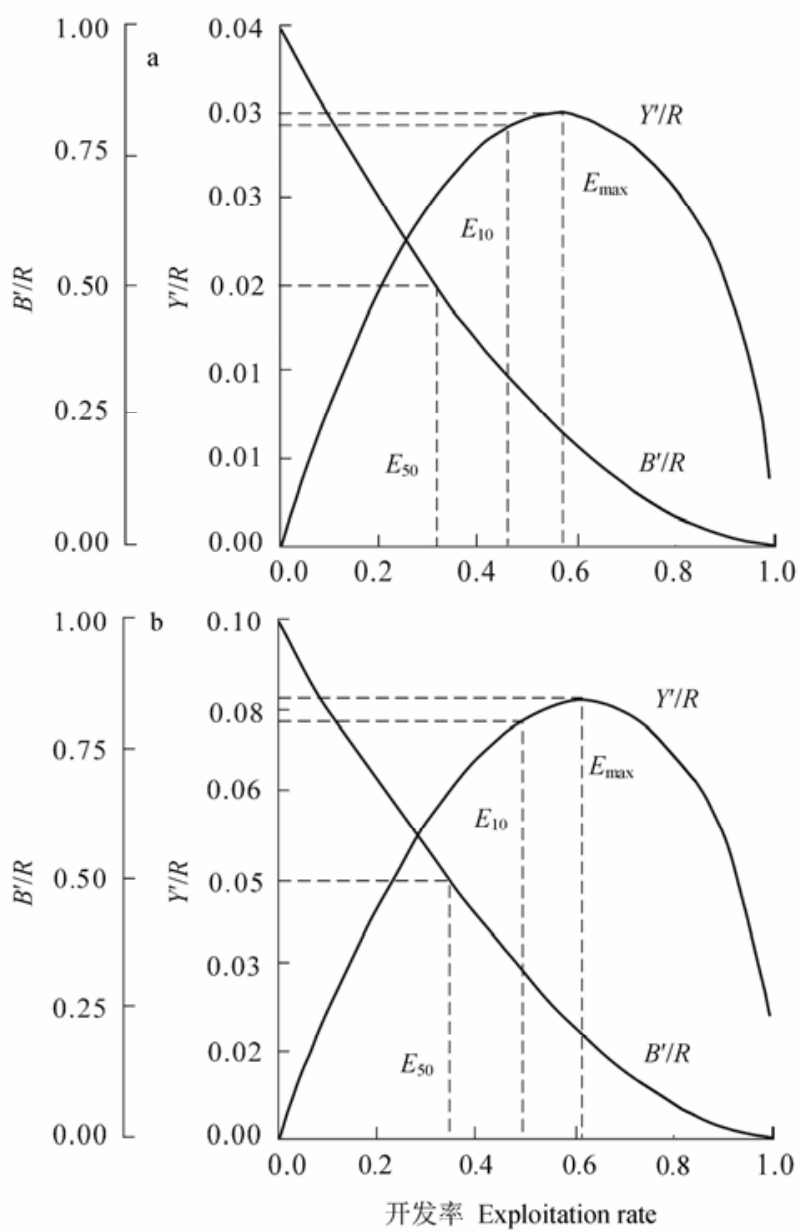

图4 江津(a)和宜宾(b)江段长鯺吻鮈的相对单位补充量产 量 $\left(Y^{\prime} / R\right)$ 和生物量 $\left(B^{\prime} / R\right)$ 曲线

Fig. 4 Relative yield per recruit $\left(Y^{\prime} / R\right)$ and biomass per recruit $\left(B^{\prime} / R\right)$ curves of Rhinogobio ventralis in the Jiangjin (a) and Yibin (b) sections

$\left.E_{\mathrm{t}}=0.8\right)$ 对长鿷吻鮈种群数量的估算结果见表 2 。与 迭代法相比, 最大体长组 $E_{\mathrm{t}}$ 取 0.5 时, 对种群尾数的 估算结果偏离 $0.7-10.2 \%$ (平均为 $4.2 \%$ ), 对种群生物 

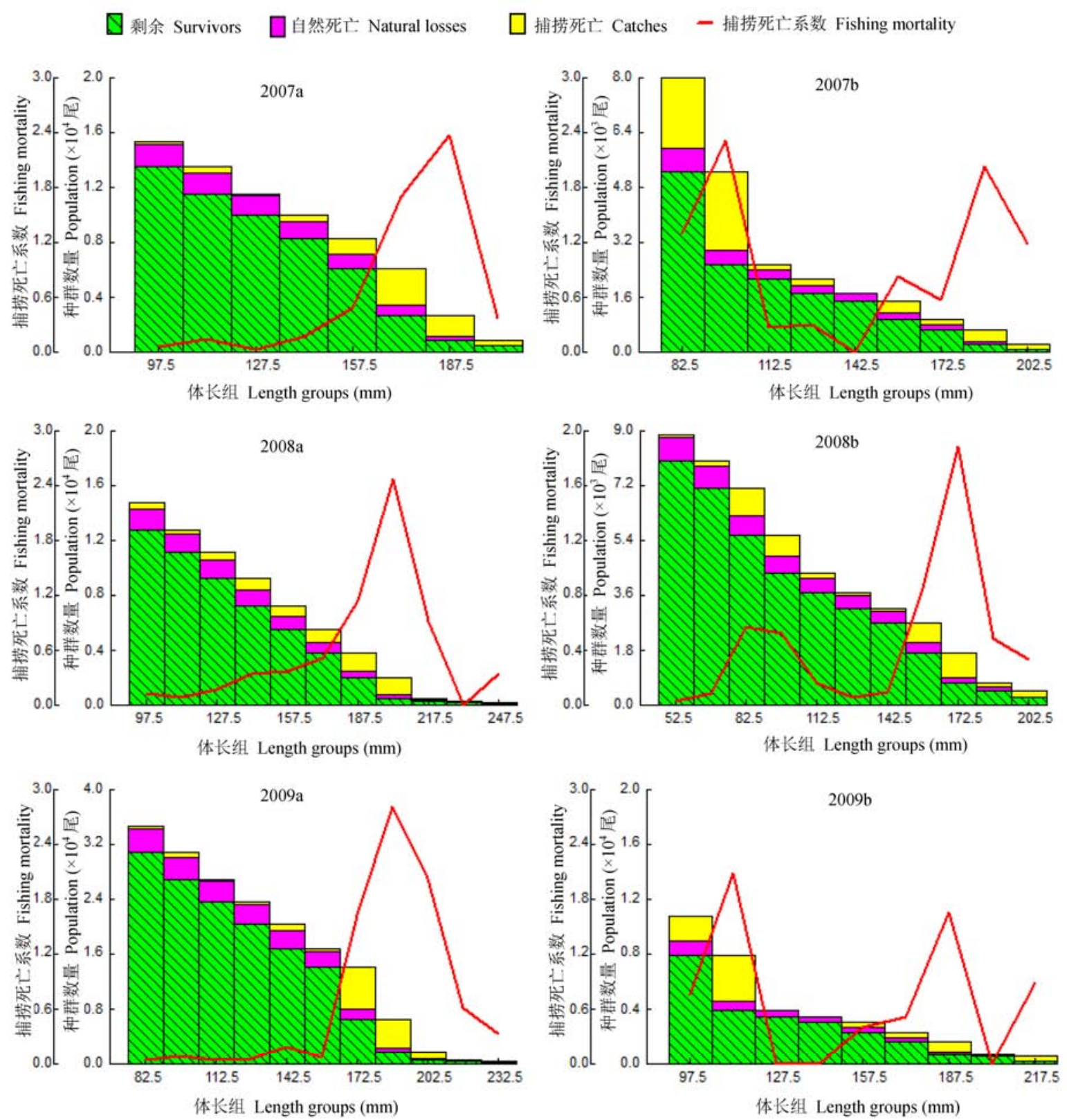

图5 2007-2009年江津(a)和宜宾(b)江段长鯺吻鮈的体长结构实际种群分析

Fig. 5 Length-structured virtual population analyses for Rhinogobio ventralis in the Jiangjin (a) and Yibin (b) sections from 2007-2009

量的估算结果偏离 $2.5-34.1 \%$ (平均为 $14.2 \%$ ); 最大 体长组 $E_{\mathrm{t}}$ 取 0.8 时, 对种群尾数的估算结果偏离 $1.5-11.9 \%$ (平均为 $5.4 \%$ ), 对种群生物量的估算结果 偏离 $5.4-34.6 \%$ (平均为 $16.3 \%$ )。

\section{3 讨论}

\section{1 种群参数和数量估算}

根据体长频率数据进行鱼类种群参数和数量 估算, 避免了因年龄鉴定的主观误差而引起的参数
评估的不准确，特别适合年龄鉴定比较困难的评估 对象，在海洋渔业资源评价中取得了较好的应用效 果(Wang et al, 2012)。本研究利用此方法估算出长鯺 吻鮈的生长参数 $L_{\infty} 、 k$ 分别为 $338 \mathrm{~mm}$ 和 $0.24 / \mathrm{yr}$ ，与以 往根据体长 - 年龄数据的估算结果相比 $\left(L_{\infty}\right.$ 为 269-316 mm, $k$ 为0.21-0.48/yr) (表3), $k$ 值位于以往 研究结果范围之内, $L_{\infty}$ 值较以往估算的最大值 316 $\mathrm{mm}$ 稍大。Froese和Binohlan (2000) 提出鱼类 $L_{\infty}$ 和其 
表2 不同资源开发率 $\left(\boldsymbol{E}_{\mathrm{t}}\right)$ 取值确定最大体长组捕捞死亡系数 $\left(\boldsymbol{F}_{\mathrm{t}}\right)$ 值时长鯺吻鮈的种群数量和生物量估算结果

Table 2 Estimate results of population abundance and biomass of Rhinogobio ventralis based on terminal fishing mortalities $\left(F_{\mathrm{t}}\right)$ determined by different exploitation rates $\left(E_{\mathrm{t}}\right)$

\begin{tabular}{|c|c|c|c|c|c|c|c|}
\hline \multirow[t]{2}{*}{ 江段 Sections } & \multirow[t]{2}{*}{ 年份 Year } & \multicolumn{3}{|c|}{ 数量 Individuals (尾) } & \multicolumn{3}{|c|}{ 生物量 Biomass (t) } \\
\hline & & 迭代法 Iteration method & $E_{\mathrm{t}}=0.5$ & $E_{\mathrm{t}}=0.8$ & 迭代法 Iteration method & $E_{\mathrm{t}}=0.5$ & $E_{\mathrm{t}}=0.8$ \\
\hline \multirow{3}{*}{$\begin{array}{l}\text { 江津 } \\
\text { Jiangjin }\end{array}$} & 2007 & 68,247 & 67,447 & 63,097 & 14.17 & 13.70 & 11.13 \\
\hline & 2008 & 67,432 & 66,719 & 64,478 & 15.55 & 15.04 & 13.46 \\
\hline & 2009 & 176,266 & 174,952 & 171,938 & 29.36 & 28.62 & 26.92 \\
\hline \multirow{3}{*}{$\begin{array}{l}\text { 宜宾 } \\
\text { Yibin }\end{array}$} & 2007 & 22,953 & 24,881 & 22,613 & 2.58 & 3.46 & 2.44 \\
\hline & 2008 & 46,340 & 44,744 & 40,832 & 5.15 & 4.62 & 3.37 \\
\hline & 2009 & 34,021 & 37,503 & 32,412 & 6.37 & 8.37 & 5.44 \\
\hline
\end{tabular}

表3 长鯺吻鮈种群参数估算结果比较

Table 3 Comparison of estimated population parameters of Rhinogobio ventralis

\begin{tabular}{|c|c|c|c|c|c|c|c|}
\hline 群体 & $\begin{array}{l}\text { 体长 } \\
\text { Length (mm) }\end{array}$ & $\begin{array}{l}L_{\infty} \\
(\mathrm{mm})\end{array}$ & $k(/ \mathrm{yr})$ & $b$ & $\begin{array}{l}\text { 样本数 } \\
\text { Number }\end{array}$ & $\begin{array}{l}\text { 估算方法 } \\
\text { Method }\end{array}$ & $\begin{array}{l}\text { 文献 } \\
\text { Reference } \\
\end{array}$ \\
\hline 乌江 Wujiang (1981-1983) & $81-260$ & - & - & 2.96 & 112 & 年龄-体长法 Age-length & Zhou \& He, 1992 \\
\hline 江津 Jiangjin (1989) & $100-280$ & 269 & 0.48 & 3.03 & 443 & 年龄-体长法 Age-length & Duan et al, 1991 \\
\hline $\begin{array}{l}\text { 长江上游 Upper Yangtze } \\
(2005-2007)\end{array}$ & River 75-236 & 299 & 0.24 & 3.21 & 546 & 年龄-体长法 Age-length & 辛建峰, 2010 \\
\hline 合江 Hejiang (1998-2001) & $81-240$ & 316 & 0.30 & 3.15 & 445 & 年龄-体长法 Age-length & 张松, $2003^{(2)}$ \\
\hline 木洞 Mudong (1998-1999) & - & 305 & 0.31 & 3.07 & 570 & 年龄-体长法 Age-length & Deng \& He, 2005 \\
\hline 丽江 Lijiang (2008) & $101-263$ & 279 & 0.33 & 3.01 & 161 & 年龄-体长法 Age-length & Bao et al, 2009 \\
\hline $\begin{array}{l}\text { 江 津 和 宜 宾 Jiangjin and } \\
\text { (2007-2009) }\end{array}$ & Yibin $52-250$ & 338 & 0.24 & 3.20 & 436 & ELEFAN I & This study \\
\hline
\end{tabular}

L市: 渐近体长; $k$ : 生长系数; $b$ : 体长-体重关系幂指数系数

$L_{\infty}$, Asymptotic length; $k$, Growth constant; $b$ : Exponent of a length-weight relationship

最小性成熟体长 $\left(L_{\mathrm{m}}\right)$ 存在经验关系，即 $\log L_{\mathrm{m}}=$ $0.8979 \log L_{\infty}-0.0782$, 长鳍吻鮈 $L_{\mathrm{m}}$ 约为 $157 \mathrm{~mm}$ (邓 辉胜和何学福, 2005), 由此估算出长鯺吻鮈的 $L_{\infty}$ 约 为 $341 \mathrm{~mm}$, 本研究 $L_{\infty}$ 估算结果与之接近。目前文献 记录的长鯺吻鮈的最大个体约为 $280 \mathrm{~mm}$ (段中华 等, 1991), 与本研究 $L_{\infty}$ 估算结果相差较大, 可能与 长鯺吻鮈常栖息于急流险滩、峡谷深潭处而难于捕 获有关。一般认为生长参数 $k$ 满足 $\mathrm{e}^{-k}<1$ 时, 用 von Bertalanffy方程能很好拟合鱼类生长, $M / k$ 值应在 1.5-2.5范围内(詹秉义, 1995), 本研究 $\mathrm{e}^{-k}=0.79, M / k$ 值为 1.8 , 在接受范围之内。

鱼类种群数量的评估方法有多种, 如标志放流 法、初级生产力估算法、鱼卵仔鱼调查法、水声学 探测法和实际种群分析法等, 其中基于体长结构的 实际种群分析法不需鉴定研究对象的年龄, 具有采 样工作量小和数据结构简单等优点而受到青崃

(1) 辛建峰 (2010) 长江上游长鳍吻鮈种群生态学研究. 硕士学位论文, 暨南大学, 广州

(2) 张松 (2003) 长江上游合江江段渔业现状评估及长鲳吻鮈的资源评 估. 硕士学位论文, 华中农业大学, 武汉.
(Gulland \& Rosenberg, 1992), 随着FiSATII渔业评 估软件的不断发展和完善, 对这一评估方法的应用 也更为高效(胡艳等, 2015)。传统的体长结构实际种 群分析中, 各体长组的 $F_{\mathrm{t}}$ 值是根据最大体长组的开 发率估算的, 不存在迭代过程(刘军等, 2010), 一般 将最大体长组 $F_{\mathrm{t}}$ 定为其自然死亡系数, 即开发率为 0.5 时的 $F_{\mathrm{t}}$, 但实际上最大体长组 $F_{\mathrm{t}}$ 可能与之差异较 大，而使用迭代法确定最大体长组的 $F_{\mathrm{t}}$, 可以提高 估算精度(吴斌等, 2013)。本研究表明, 最大体长组 $E_{\mathrm{t}}$ 取值 0.5 和 0.8 时, 种群数量估算结果与迭代法确 定 $F_{\mathrm{t}}$ 的估算结果平均偏离约 $5 \%$, 而种群生物量的估 算结果相差较大, 平均偏离约 $15 \%$ 。

\section{2 种群现状与保护}

辛建峰等(2010)研究显示, 长江干流不同江段 长鯺吻鮈个体大小差异显著, 下游江段个体大于上 游江段, 本研究结果与之一致, 下游江津江段长鯺 吻鮈个体明显大于上游宜宾江段。 20 世纪 80 年代末, 江津江段长鯺吻鮈平均体长约 $164.1 \mathrm{~mm}$, 优势体长 组为 100-180 mm(段中华等, 1991), 本研究显示, 2007-2009年该江段长鯺吻鮈的平均体长为 169.0 
$\mathrm{mm}$, 优势体长组为 135-210 $\mathrm{mm}$, 两时期的体长结 构基本相似。三峡水库蓄水前(1998-2002年), 宜宾 江段长鯺吻鮈的种群数量在 $1,294-4,421$ 尾 $/ \mathrm{km}$ 范围 波动, 平均种群数量为 2,506 尾 $/ \mathrm{km}$ (刘军等, 2010), 本研究结果与之相近, 2007-2009年长鯺吻鮈种群 数量在1,530-3,089尾 $/ \mathrm{km}$ 范围波动, 平均种群数量 为 2,296 尾 $/ \mathrm{km}$, 表明三峡水库蓄水前后宜宾江段长 鯺吻鮈种群数量相对稳定。合江江段长鯺吻鮈的种 群数量为 5,726 尾 $/ \mathrm{km}^{\circledR}$, 本研究显示江津和宜宾江 段长鰙吻鮈的平均种群数量分别为 6,932 和 2,296 尾 $/ \mathrm{km}$, 表明下游江段的长鯺吻鮈种群数量要高于 上游江段。三峡水库从2003年开始蓄水, 长鯺吻鮈 等流水性种类逐渐从库区迁移到上游流水江段, 江 津江段靠近三峡水库库区, 鱼类种群可能受水库调 节影响较大。2009年长鯺吻鮈种群数量突然升高, 可能与三峡水库的水位调节有关。

长鯺吻鮈仍是长江上游的主要渔获对象之一。 Froese和Binohlan (2000)提出能获得最大相对渔获 量的最适捕捞体长 $\left(L_{\mathrm{opt}}\right)$ 可由最小性成熟体长 $L_{\mathrm{m}}$ 估 算, 即 $\log L_{\mathrm{opt}}=1.053 \log L_{\mathrm{m}}-0.0565$ 。长鯺吻鮈 $L_{\mathrm{m}}$ 为 $157 \mathrm{~mm}$ (邓辉胜和何学福, 2005), 由此估算出其最 适开捕体长为 $180.2 \mathrm{~mm}$ 。本研究表明, 目前江津和 宜宾江段渔获群体的平均体长为 $150.8 \mathrm{~mm}$, 远低于 最适开捕体长。江津和宜宾江段长鯺吻鮈的资源开 发率分别为 0.81 和 0.79 , 均已超过了其最大开发率, 属过度开发。

随着三峡、向家坝和溪洛渡等大型梯级水利工 程的相继建设, 长江上游鱼类栖息生境已支离破 碎, 适应流水生境的长鯺吻鮈的栖息生境严重萎 缩, 坝上和坝下种群也得不到有效交流。长鯺吻鮈 产漂流性卵, 必须要保证足够的漂流距离才能发育 成具有主动游泳能力的鱼苗, 这些梯级电站的建 设, 将影响其产卵场的分布及卵、苗的发育, 从而 影响其种群生存(段辛斌等, 2015)。长江上游长鯺吻 鮈种群生存受到严重威胁(刘军, 2004)。建议在春季 禁渔的基础上, 延长禁渔期, 养护渔业资源。加强 长鯺吻鮈种群动态监测及重要栖息生境的保护和 修复。本研究显示, 江津等下游江段的长鯺吻鮈种 群数量要大于上游江段, 应重点加强江津等下游江 段的长鯺吻鮈种群保护。

(1) 张松 (2003) 长江上游合江江段渔业现状评估及长鳍吻鮈的资源评 估. 硕士学位论文, 华中农业大学, 武汉.

\section{参考文献}

Bao XG, Xie WX, Huang DM, Zhu BK (2009) Study on the age and growth of Rhinogobio ventralis in Jinsha River. Journal of Anhui Agricultural Science, 37, 10017-10019. (in Chinese with English abstract) [鲍新国, 谢文星, 黄道明, 朱邦科 (2009) 金沙江长鯺吻鮈年龄与生长的研究. 安 徽农业科学, 37, 10017-10019.]

Chen DQ, Chang JB, Gu HB (2005) Impacts of Jinsha River first stage project on ecology and environment of natural reserve and its countermeasures. Journal of Yangtze River Scientific Research Institute, 22(2), 21-24. (in Chinese with English abstract) [陈大庆, 常剑波, 顾洪宾 (2005) 金沙 江一期工程对保护区生态环境的影响与对策. 长江科学 院院报, 22(2), 21-24.]

Deng HS, He XF (2005) Study on the biology of Rhinogobio ventralis in the Changjiang River. Journal of Southwest Agricultural University (Natural Science), 27, 704-708. (in Chinese with English abstract) [邓辉胜, 何学福 (2005) 长 江干流长鯺吻鮈的生物学研究. 西南农业大学学报(自然 科学版), 27, 704-708.]

Ding RH (1994) The Fishes of Sichuan. Sichuan Press of Science and Technology, Chengdu. (in Chinese) [丁瑞华 (1994) 四川鱼类志. 四川科学技术出版社, 成都.]

Duan XB, Tian HW, Gao TH, Liu SP, Wang K, Chen DQ (2015) Resources status of ichthyoplankton in the upper Yangtze River before the storage of Jinsha River first stage project. Resources and Environment in the Yangtze Basin, 24, 1358-1365. (in Chinese with English abstract) [段辛斌, 田辉伍, 高天珩, 刘绍平, 王珂, 陈大庆 (2015) 金沙江 一期工程蓄水前长江上游产漂流性卵鱼类产卵场现状. 长江流域资源与环境, 24, 1358-1365.]

Duan ZH, Chang JB, Sun JY (1991) Age and growth of Rhinogobio ventralis in Changjiang River. Freshwater Fisheries, 21(2), 12-14. (in Chinese with English abstract) [段中 华, 常剑波, 孙建贻 (1991) 长鯺吻鮈年龄和生长的研 究. 淡水渔业, 21(2), 12-14.]

Esguicero ALH, Arcifa M (2010) Fragmentation of a neotropical migratory fish population by a century-old dam. Hydrobiologia, 638, 41-53.

Fahrig L (2003) Effects of habitat fragmentation on biodiversity. Annual Review of Ecology, Evolution, and Systematics, 34, 487-515.

Froese R, Binohlan C (2000) Empirical relationships to estimate asymptotic length, length at first maturity and length at maximum yield per recruit in fishes, with a simple method to evaluate length frequency data. Journal of Fish Biology, $56,758-773$.

Gao X, Zeng Y, Wang JW, Liu HZ (2010) Immediate impacts of the second impoundment on fish communities in the Three Gorges Reservoir. Environmental Biology of Fishes, 87, 163-173.

Gayanilo FC Jr, Sparre P, Pauly D (2005) FAO-ICLARM Stock Assessment Tools II (User's Guide). Worldfish Center, FAO, Rome. 
Ghosh S, Sivadas M, Abdussamad EM, Rohit P, Koya KPS, Joshi KK, Chellappan A, Rathinam MM, Prakasan D, Sebastine M (2012) Fishery, population dynamics and stock structure of frigate tuna Auxis thazard (Lacepede, 1800) exploited from Indian waters. Indian Journal of Fisheries, 59, 95-100.

Gulland JA, Rosenberg AA (1992) A Review of Length-based Approaches to Assessing Fish Stocks. FAO, Rome.

Gunderson DR, Dygert PH (1988) Reproductive effort as a predictor of natural mortality rate. ICES Journal of Marine Science, 44, 200-209.

Hu Y, Zhang T, Yang G, Zhao F, Hou JL, Zhang LZ, Zhuang P (2015) Assessment of resource situation of Collichthys lucidus in coastal waters of the Yangtze estuary. Chinese Journal of Applied Ecology, 26, 2867-2873. (in Chinese with English abstract) [胡艳, 张涛, 杨刚, 赵峰, 侯俊利, 章龙珍, 庄平 (2015) 长江口近岸水域棘头梅童鱼资源 现状的评估. 应用生态学报, 26, 2867-2873.]

Jensen AL (1996) Beverton and Holt life history invariants result from optimal trade-off of reproduction and survival. Canadian Journal of Fisheries and Aquatic Sciences, 53, $820-822$.

Jiang ZG, Ge S (2005) Exploring the mechanism of species endangerment and conservation strategy in the Yangtze River drainage. Biodiversity Science, 13, 367-375. (in Chinese with English abstract) [蒋志刚, 葛颂 (2005) 探索长江流域物种 濒危机制与保护对策. 生物多样性, 13, 367-375.]

Johnson MG, Tamatamah AR (2013) Length frequency distribution, mortality rate and reproduction biology of Kawakama (Euthynnus affinis-Cantor, 1849) in the coastal waters of Tanzania. Pakistan Journal of Biological Sciences, 16, $1270-1278$.

Keys AB (1928) The weight-length relationship in fishes. Proceedings of the National Academy of Sciences, USA, 14, 922-925.

Letcher BH, Nislow KH, Coombs JA, O’Donnell MJ, Dubreuil TL (2007) Population response to habitat fragmentation in a stream-dwelling brook trout population. PLoS ONE, 11, $1-11$.

Liu J (2004) A quantitative analysis on threat and priority of conservation order of the endemic fishes in upper reaches of the Yangtze River. China Environmental Science, 24, 395-399. (in Chinese with English abstract) [刘军 (2004) 长江上游特有鱼类受威胁及优先保护顺序的定量分析. 中国环境科学, 24, 395-399.]

Liu J, Wang JW, Miao ZG, Cao WX (2010) Estimation on abundance of Rhinogobio ventralis in Yibin reach of the Yangtze River. Resources and Environment in the Yangtze Basin, 19, 276-280. (in Chinese with English abstract) [刘 军, 王剑伟, 苗志国, 曹文宣 (2010) 长江上游宜宾江段 长鯺吻鮈种群资源量的估算. 长江流域资源与环境, 19 , 276-280.]

Morita K, Morita SH, Yamamoto S (2009) Effects of habitat fragmentation by damming on salmonid fishes, lessons from white-spotted charr in Japan. Ecological Research, 24,
711-722.

Park YS, Chang JB, Lek S, Cao WX, Brosse S (2003) Conservation strategies for endemic fish species threatened by the Three Gorges Dam. Conservation Biology, 17, 1748-1758.

Pauly D (1979) Gill size and temperature as governing factors in fish growth, a generalization of von Bertalanffy's growth formula. Berichte aus dem Institut fuer Meereskunde an der Christian-Albrechts-Universitaet, Kiel, Germany.

Pauly D (1980) On the interrelationships between natural mortality, growth parameters, and mean environmental temperature in 175 fish stocks. ICES Journal of Marine Science, 39, 175-192.

Tian HW, Duan XB, Xiong X, Luo HW, Liu SP, Chen DQ (2013) Estimation of growth and population parameters of Elongate Loach (Leptobotia elongate) in the upper reaches of the Yangtze River. Resources and Environment in the Yangtze Basin, 22, 1305-1312. (in Chinese with English abstract) [田辉伍, 段辛斌, 熊星, 罗宏伟, 刘绍平, 陈大 庆 (2013) 长江上游长薄鳅生长和种群参数的估算. 长 江流域资源与环境, 22, 1305-1312.]

Wang XH, Qiu YS, Du FY, Lin ZJ, Sun DR, Huang SL (2012) Population parameters and dynamic pool models of commercial fishes in the Beibu Gulf, northern South China Sea. Chinese Journal of Oceanology and Limnology, 30, 105-117.

Wu B, Fang CL, He G, Fu PF (2013) FiSATII software supported length based cohort analysis. South China Fisheries Sciences, 9(4), 94-98. (in Chinese with English abstract) [吴 斌, 方春林, 贺刚, 傅培峰 (2013) FiSATII软件支持下的 体长股分析法探讨. 南方水产科学, 9(4), 94-98.]

Xie P (2003) Three-Gorges Dam: risk to ancient fish. Science, $302,1149$.

Xin JF, Yang YF, Liu HZ (2010) Study on the age and growth of Rhinogobio ventralis in the upper reaches of the Yangtze River. Sichuan Journal of Zoology, 29, 352-356. (in Chinese with English abstract) [辛建峰, 杨宇峰, 刘焕章 (2010) 长江上游长鯺吻鮈年龄与生长的研究. 四川动物, 29, 352-356.]

Xiong F, Liu HY, Duan XB, Liu SP, Chen DQ (2014) Community structure of fish and resources utilization in Jiangjin section of the upper Yangtze River. Journal of Anhui University (Natural Sciences), 38(3), 94-102. (in Chinese with English abstract) [熊飞, 刘红艳, 段辛斌, 刘绍平, 陈大庆 (2014) 长江上游江津江段鱼类群落结构及资源利用. 安 徽大学学报(自然科学版), 38(3), 94-102.]

Zhan BY (1995) Fish Stock Assessment. China Agriculture Press, Beijing. (in Chinese) [詹秉义 (1995) 渔业资源评 估. 中国农业出版社, 北京.]

Zhou QG, He XF (1992) A preliminary study on biology of Rhinogobio ventralis in Wujiang River. Freshwater Fisheries, 22(5), 11-14. (in Chinese with English abstract) [周启 贵, 何学福 (1992) 长鯺吻鮈生物学的初步研究. 淡水渔 业, 22(5), 11-14.]

(责任编委: 陈小勇 责任编辑: 问文杰) 